\title{
Oscillations of Difference Equations with Several Oscillating Coefficients
}

\author{
L. Berezansky, ${ }^{1}$ G. E. Chatzarakis, ${ }^{2}$ A. Domoshnitsky, ${ }^{3}$ and I. P. Stavroulakis ${ }^{4}$ \\ ${ }^{1}$ Department of Mathematics, Ben-Gurion University of Negev, 84105 Beer-Sheva, Israel \\ ${ }^{2}$ Department of Electrical and Electronic Engineering Educators, School of Pedagogical and Technological Education (ASPETE), \\ N. Heraklion, 14121 Athens, Greece \\ ${ }^{3}$ Department of Mathematics and Computer Sciences, Ariel University of Samaria, 40700 Ariel, Israel \\ ${ }^{4}$ Department of Mathematics, University of Ioannina, 45110 Ioannina, Greece
}

Correspondence should be addressed to A. Domoshnitsky; adom@ariel.ac.il

Received 18 April 2014; Accepted 10 May 2014; Published 1 June 2014

Academic Editor: Tongxing Li

Copyright (C) 2014 L. Berezansky et al. This is an open access article distributed under the Creative Commons Attribution License, which permits unrestricted use, distribution, and reproduction in any medium, provided the original work is properly cited.

We study the oscillatory behavior of the solutions of the difference equation $\Delta x(n)+\sum_{i=1}^{m} p_{i}(n) x\left(\tau_{i}(n)\right)=0, n \in \mathbb{N}_{0}[\nabla x(n)-$ $\left.\sum_{i=1}^{m} p_{i}(n) x\left(\sigma_{i}(n)\right)=0, n \in \mathbb{N}\right]$ where $\left(p_{i}(n)\right), 1 \leq i \leq m$ are real sequences with oscillating terms, $\tau_{i}(n)\left[\sigma_{i}(n)\right], 1 \leq i \leq m$ are general retarded (advanced) arguments, and $\Delta[\nabla]$ denotes the forward (backward) difference operator $\Delta x(n)=x(n+1)-$ $x(n)[\nabla x(n)=x(n)-x(n-1)]$. Examples illustrating the results are also given.

\section{Introduction}

In the present paper, we study the oscillatory behavior of the solutions of the difference equation

$$
\Delta x(n)+\sum_{i=1}^{m} p_{i}(n) x\left(\tau_{i}(n)\right)=0, \quad n \in \mathbb{N}_{0},
$$

where $\mathbb{N} \ni m \geq 2, p_{i}, 1 \leq i \leq m$ are real sequences with oscillating terms, and $\left\{\tau_{i}(n)\right\}_{n \in \mathbb{N}_{0}}, 1 \leq i \leq m$ are sequences of integers such that

$$
\tau_{i}(n) \leq n-1, \quad n \in \mathbb{N}_{0}, \quad \lim _{n \rightarrow \infty} \tau_{i}(n)=\infty, \quad 1 \leq i \leq m
$$

and the (dual) advanced difference equation

$$
\nabla x(n)-\sum_{i=1}^{m} p_{i}(n) x\left(\sigma_{i}(n)\right)=0, \quad n \in \mathbb{N},
$$

where $\mathbb{N} \ni m \geq 2, p_{i}, 1 \leq i \leq m$ are real sequences with oscillating terms and $\left\{\sigma_{i}(n)\right\}_{n \in \mathbb{N}}, 1 \leq i \leq m$, are sequences of integers such that

$$
\sigma_{i}(n) \geq n+1, \quad n \in \mathbb{N}, \quad 1 \leq i \leq m .
$$

Here, $\mathbb{N}_{0}=\{0,1,2, \ldots\}$ and $\mathbb{N}=\{1,2, \ldots\}$. Also, as usual, $\Delta$ denotes the forward difference operator $\Delta x(n)=x(n+1)-$ $x(n)$ and $\nabla$ denotes the backward difference operator $\nabla x(n)=$ $x(n)-x(n-1)$.

Strong interest in $\left(\mathrm{E}_{\mathrm{R}}\right)$ is motivated by the fact that it represents a discrete analogue of the differential equation (see [1] and the references cited therein)

$$
x^{\prime}(t)+\sum_{i=1}^{m} p_{i}(t) x\left(\tau_{i}(t)\right)=0, \quad t \geq 0,
$$

where, for every $i \in\{1, \ldots, m\}, p_{i}$ is an oscillating continuous real-valued function in the interval $[0, \infty)$, and $\tau_{i}$ is a continuous real-valued function on $[0, \infty)$ such that

$$
\tau_{i}(t) \leq t, \quad t \geq 0, \quad \lim _{t \rightarrow \infty} \tau_{i}(t)=\infty,
$$

while, $\left(\mathrm{E}_{\mathrm{A}}\right)$ represents a discrete analogue of the advanced differential equation (see [1] and the references cited therein)

$$
x^{\prime}(t)-\sum_{i=1}^{m} p_{i}(t) x\left(\sigma_{i}(t)\right)=0, \quad t \geq 1,
$$


where, for every $i \in\{1, \ldots, m\}, p_{i}$ is an oscillating continuous real-valued function in the interval $[1, \infty)$ and $\sigma_{i}$ is a continuous real-valued function on $[1, \infty)$ such that

$$
\sigma_{i}(t) \geq t, \quad t \geq 1
$$

By a solution of $\left(\mathrm{E}_{\mathrm{R}}\right)$, we mean a sequence of real numbers $\{x(n)\}_{n \geq-w}$ which satisfies $\left(\mathrm{E}_{\mathrm{R}}\right)$ for all $n \in \mathbb{N}_{0}$. Here,

$$
w=-\min _{n \geq 0}\left\{\tau_{i}(n): 1 \leq i \leq m\right\}
$$

It is clear that, for each choice of real numbers $c_{-w}, c_{-w+1}, \ldots, c_{-1}, c_{0}$, there exists a unique solution $\{x(n)\}_{n \geq-w}$ of $\left(\mathrm{E}_{\mathrm{R}}\right)$ which satisfies the initial conditions $x(-w)=c_{-w}$, $x(-w+1)=c_{-w+1}, \ldots, x(-1)=c_{-1}$, and $x(0)=c_{0}$.

By a solution of the advanced difference equation $\left(\mathrm{E}_{\mathrm{A}}\right)$, we mean a sequence of real numbers $\{x(n)\}_{n \in \mathbb{N}_{0}}$ which satisfies $\left(\mathrm{E}_{\mathrm{A}}\right)$ for all $n \in \mathbb{N}$.

A solution $\{x(n)\}_{n \geq-w}\left[\{x(n)\}_{n \in \mathbb{N}_{0}}\right]$ of $\left(\mathrm{E}_{\mathrm{R}}\right)\left[\left(\mathrm{E}_{\mathrm{A}}\right)\right]$ is called oscillatory, if the terms $x(n)$ of the sequence are neither eventually positive nor eventually negative. Otherwise, the solution is said to be nonoscillatory.

In the last few decades, the oscillatory behavior of all solutions of difference equations has been extensively studied when the coefficients $p_{i}(n)$ are nonnegative. See, for example, [2-20] and the references cited therein. However, for the general case when $p_{i}(n)$ are allowed to oscillate, it is difficult to study the oscillation of $\left(\mathrm{E}_{\mathrm{R}}\right)\left[\left(\mathrm{E}_{\mathrm{A}}\right)\right]$, since the difference $\Delta x(n)[\nabla x(n)]$ of any nonoscillatory solution of $\left(\mathrm{E}_{\mathrm{R}}\right)\left[\left(\mathrm{E}_{\mathrm{A}}\right)\right]$ is always oscillatory. Thus, a small number of papers are dealing with this case. See, for example, $[1,21-32]$ and the references cited therein.

For (3) and (5) with oscillating coefficients, Fukagai and Kusano [1] established the following theorems.

Theorem 1 (see [1, Theorem $\left.3^{\prime}(\mathrm{i})\right]$ ). Assume (4) and that there is a continuous nondecreasing function $\tau^{*}(t)$ such that $\tau_{i}(t) \leq$ $\tau^{*}(t) \leq t$ for $t \geq 0,1 \leq i \leq m$. Suppose moreover that there is a sequence $\left\{t_{n}\right\}_{n \in \mathbb{N}}$ such that $\lim _{n \rightarrow \infty} t_{n}=\infty$, the intervals $\bigcup_{n \in \mathbb{N}}\left[\left(\tau^{*}\right)^{n}\left(t_{n}\right), t_{n}\right]$ are disjoint, and

$$
p_{i}(t) \geq 0 \quad \forall t \in \bigcup_{n \in \mathbb{N}}\left[\left(\tau^{*}\right)^{n}\left(t_{n}\right), t_{n}\right], 1 \leq i \leq m .
$$

If there is a constant $c$ such that

$$
\int_{\tau^{*}(t)}^{t} \sum_{i=1}^{m} p_{i}(s) d s>c>\frac{1}{e} \quad \forall t \in \bigcup_{n \in \mathbb{N}}\left[\left(\tau^{*}\right)^{n-1}\left(t_{n}\right), t_{n}\right],
$$

then all solutions of (3) oscillate.

Theorem 2 (see [1, Theorem $3^{\prime}$ (ii)]). Assume (6) and that there is a continuous nondecreasing function $\sigma_{*}(t)$ such that $t \leq \sigma_{*}(t) \leq \sigma_{i}(t)$ for $t \geq 0,1 \leq i \leq m$. Suppose moreover that there is a sequence $\left\{t_{n}\right\}_{n \in \mathbb{N}}$ such that $\lim _{n \rightarrow \infty} t_{n}=\infty$, the intervals $\bigcup_{n \in \mathbb{N}}\left[t_{n},\left(\sigma_{*}\right)^{n}\left(t_{n}\right)\right]$ are disjoint, and

$$
p_{i}(t) \geq 0 \quad \forall t \in \bigcup_{n \in \mathbb{N}}\left[t_{n},\left(\sigma_{*}\right)^{n}\left(t_{n}\right)\right], 1 \leq i \leq m .
$$

If there is a constant $c$ such that

$$
\int_{t}^{\sigma_{*}(t)} \sum_{i=1}^{m} p_{i}(s) d s>c>\frac{1}{e} \quad \forall t \in \bigcup_{n \in \mathbb{N}}\left[t_{n},\left(\sigma_{*}\right)^{n-1}\left(t_{n}\right)\right],
$$

then all solutions of (5) oscillate.

For $\left(\mathrm{E}_{\mathrm{R}}\right)$ and $\left(\mathrm{E}_{\mathrm{A}}\right)$ with oscillating coefficients, recently, Bohner et al. [21, 23] established the following theorems.

Theorem 3 (see [23, Theorem 2.4]). Assume (1) and that the sequences $\tau_{i}$ are increasing for all $i \in\{1, \ldots, m\}$. Suppose also that for each $i \in\{1, \ldots, m\}$ there exists a sequence $\left\{n_{i}(j)\right\}_{j \in \mathbb{N}}$ such that $\lim _{j \rightarrow \infty} n_{i}(j)=\infty$ and

$$
\begin{array}{r}
p_{k}(n) \geq 0 \quad \forall n \in \bigcap_{i=1}^{m}\left\{\bigcup_{j \in \mathbb{N}}\left[\tau\left(\tau\left(n_{i}(j)\right)\right), n_{i}(j)\right] \cap \mathbb{N}\right\} \neq \emptyset, \\
1 \leq k \leq m,
\end{array}
$$

where

$$
\tau(n)=\max _{1 \leq i \leq m} \tau_{i}(n), \quad n \in \mathbb{N}_{0}
$$

If, moreover,

$$
\limsup _{j \rightarrow \infty} \sum_{i=1}^{m} \sum_{q=\tau(n(j))}^{n(j)} p_{i}(q)>1
$$

where $n(j)=\min \left\{n_{i}(j): 1 \leq i \leq m\right\}$, then all solutions of $\left(\mathrm{E}_{\mathrm{R}}\right)$ oscillate.

Theorem 4 (see [23, Theorem 3.4]). Assume (2) and that the sequences $\sigma_{i}$ are increasing for all $i \in\{1, \ldots, m\}$. Suppose also that for each $i \in\{1, \ldots, m\}$ there exists a sequence $\left\{n_{i}(j)\right\}_{j \in \mathbb{N}}$ such that $\lim _{j \rightarrow \infty} n_{i}(j)=\infty$ and

$$
\begin{array}{r}
p_{k}(n) \geq 0 \quad \forall n \in \bigcap_{i=1}^{m}\left\{\bigcup_{j \in \mathbb{N}}\left[n_{i}(j), \sigma\left(\sigma\left(n_{i}(j)\right)\right)\right] \cap \mathbb{N}\right\} \neq \emptyset, \\
1 \leq k \leq m,
\end{array}
$$

where

$$
\sigma(n)=\min _{1 \leq i \leq m} \sigma_{i}(n), \quad n \in \mathbb{N}
$$

If, moreover,

$$
\limsup _{j \rightarrow \infty} \sum_{i=1}^{m} \sum_{q=n(j)}^{\sigma(n(j))} p_{i}(q)>1,
$$

where $n(j)=\max \left\{n_{i}(j): 1 \leq i \leq m\right\}$, then all solutions of $\left(\mathrm{E}_{\mathrm{A}}\right)$ oscillate. 
Theorem 5 (see [21, Theorem 2.1]). Assume (1) and that the sequences $\tau_{i}$ are increasing for all $i \in\{1, \ldots, m\}$. Suppose also that for each $i \in\{1, \ldots, m\}$ there exists a sequence $\left\{n_{i}(j)\right\}_{j \in \mathbb{N}}$ such that $\lim _{j \rightarrow \infty} n_{i}(j)=\infty$,

$$
p_{k}(n) \geq 0 \quad \forall n \in \bigcap_{i=1}^{m}\left\{\bigcup_{j \in \mathbb{N}}\left[\tau_{i}\left(\tau_{i}\left(n_{i}(j)\right)\right), n_{i}(j)\right] \cap \mathbb{N}\right\} \neq \emptyset
$$$$
1 \leq k \leq m
$$

$\limsup _{n \rightarrow \infty} \sum_{i=1}^{m} p_{i}(n)>0$

$\forall n \in \bigcap_{i=1}^{m}\left\{\bigcup_{j \in \mathbb{N}}\left[\tau_{i}\left(\tau_{i}\left(n_{i}(j)\right)\right), n_{i}(j)\right] \cap \mathbb{N}\right\}$

If, moreover,

$$
\liminf _{j \rightarrow \infty} \sum_{i=1}^{m} \sum_{q=\tau_{i}\left(n_{i}(j)\right)}^{n_{i}(j)-1} p_{i}(q)>\frac{1}{e}
$$

then all solutions of $\left(\mathrm{E}_{\mathrm{R}}\right)$ oscillate.

Theorem 6 (see [21, Theorem 3.1]). Assume (2) and that the sequences $\sigma_{i}$ are increasing for all $i \in\{1, \ldots, m\}$. Suppose also that for each $i \in\{1, \ldots, m\}$ there exists a sequence $\left\{n_{i}(j)\right\}_{j \in \mathbb{N}}$ such that $\lim _{j \rightarrow \infty} n_{i}(j)=\infty$,

$$
\begin{array}{r}
p_{k}(n) \geq 0 \quad \forall n \in \bigcap_{i=1}^{m}\left\{\bigcup_{j \in \mathbb{N}}\left[n_{i}(j), \sigma_{i}\left(\sigma_{i}\left(n_{i}(j)\right)\right)\right] \cap \mathbb{N}\right\} \neq \emptyset, \\
1 \leq k \leq m,
\end{array}
$$

$\limsup _{n \rightarrow \infty} \sum_{i=1}^{m} p_{i}(n)>0$

$\forall n \in \bigcap_{i=1}^{m}\left\{\bigcup_{j \in \mathbb{N}}\left[n_{i}(j), \sigma_{i}\left(\sigma_{i}\left(n_{i}(j)\right)\right)\right] \cap \mathbb{N}\right\}$

If, moreover,

$$
\liminf _{j \rightarrow \infty} \sum_{i=1}^{m} \sum_{q=n_{i}(j)+1}^{\sigma_{i}\left(n_{i}(j)\right)} p_{i}(q)>\frac{1}{e}
$$

then all solutions of $\left(\mathrm{E}_{\mathrm{A}}\right)$ oscillate.

In the present paper, the authors study further $\left(\mathrm{E}_{\mathrm{R}}\right)$ $\left[\left(\mathrm{E}_{\mathrm{A}}\right)\right]$ and derive new sufficient oscillation conditions when neither (14) [(17)] nor (20) [(23)] is satisfied (cf. [6-8] and the references cited therein in the case of the equations $\left(\mathrm{E}_{\mathrm{R}}\right)$ $\left[\left(\mathrm{E}_{\mathrm{A}}\right)\right]$ with nonnegative coefficients $\left.p_{i}, 1 \leq i \leq m\right)$. Examples illustrating the results are also given.

\section{Retarded Equations}

In this section, we present new sufficient conditions for the oscillation of all solutions of $\left(\mathrm{E}_{\mathrm{R}}\right)$ when the conditions (14) and (20) are not satisfied, under the assumption that the sequences $\tau_{i}$ are increasing for all $i \in\{1, \ldots, m\}$. To that end, the following lemma provides a useful tool.

Lemma 7. Assume that (1) holds, the sequences $\tau_{i}$ are increasing for all $i \in\{1, \ldots, m\}$ and $(x(n))_{n \geq-w}$ is a nonoscillatory solution of $\left(\mathrm{E}_{\mathrm{R}}\right)$. Suppose also that for each $i \in\{1, \ldots, m\}$ there exists a sequence $\left\{n_{i}(j)\right\}_{j \in \mathbb{N}}$, such that $\lim _{j \rightarrow \infty} n_{i}(j)=\infty$, and (12) where $\tau$ is defined by (13). Set

$$
\alpha:=\liminf _{j \rightarrow \infty} \sum_{i=1}^{m} \sum_{q=\tau(n(j))}^{n(j)-1} p_{i}(q)
$$

where $n(j)=\min \left\{n_{i}(j): 1 \leq i \leq m\right\}$.

If $0<\alpha<1$, then

$$
\liminf _{j \rightarrow \infty} \frac{x(n(j)+1)}{x(\tau(n(j)))} \geq \frac{\alpha^{2}}{4(1-\alpha)} .
$$

Proof. Since the solution $\{x(n)\}_{n \geq-w}$ of $\left(\mathrm{E}_{\mathrm{R}}\right)$ is nonoscillatory, it is either eventually positive or eventually negative. As $\{-x(n)\}_{n \geq-w}$ is also a solution of $\left(\mathrm{E}_{\mathrm{R}}\right)$, we may restrict ourselves only to the case where $x(n)>0$ eventually.

By (12), it is obvious that there exists $j_{0} \in \mathbb{N}$ such that

$$
p_{k}(n) \geq 0 \quad \forall n \in \bigcap_{i=1}^{m}\left[\tau\left(\tau\left(n_{i}\left(j_{0}\right)\right)\right), n_{i}\left(j_{0}\right)\right] \cap \mathbb{N},
$$

$$
x\left(\tau_{k}(n)\right)>0 \quad \forall n \in \bigcap_{i=1}^{m}\left[\tau\left(\tau\left(n_{i}\left(j_{0}\right)\right)\right), n_{i}\left(j_{0}\right)\right] \cap \mathbb{N}
$$

$$
1 \leq k \leq m
$$

Also, by (24) we have

$$
\sum_{i=1}^{m} \sum_{q=\tau\left(n\left(j_{0}\right)\right)}^{n\left(j_{0}\right)-1} p_{i}(q) \geq \alpha-\varepsilon
$$

where $\varepsilon$ is an arbitrary real number with $0<\varepsilon<\alpha$.

In view of (26) and (27), $\left(E_{R}\right)$ gives

$$
x(n+1)-x(n)=-\sum_{i=1}^{m} p_{i}(n) x\left(\tau_{i}(n)\right) \leq 0,
$$

for every $n \in \bigcap_{i=1}^{m}\left[\tau\left(\tau\left(n_{i}\left(j_{0}\right)\right)\right), n_{i}\left(j_{0}\right)\right] \cap \mathbb{N}$. This guarantees that the sequence $x$ is decreasing on $\bigcap_{i=1}^{m}\left[\tau\left(\tau\left(n_{i}\left(j_{0}\right)\right)\right)\right.$, $\left.n_{i}\left(j_{0}\right)\right] \cap \mathbb{N}$.

Assume that $0<\alpha<1$, where $\alpha$ is defined by (24). From inequality (28), it is clear that there exists $n^{*}\left(j_{0}\right) \geq n\left(j_{0}\right)$ such that

$$
\sum_{i=1}^{m} \sum_{q=n\left(j_{0}\right)}^{n^{*}\left(j_{0}\right)-1} p_{i}(q)<\frac{\alpha-\varepsilon}{2}, \quad \sum_{i=1}^{m} \sum_{q=n\left(j_{0}\right)}^{n^{*}\left(j_{0}\right)} p_{i}(q) \geq \frac{\alpha-\varepsilon}{2} .
$$


This is because in the case where $p_{i}(q)<(\alpha-\varepsilon) / 2$, there exists $n^{*}\left(j_{0}\right)>n\left(j_{0}\right)$ such that $(30)$ is satisfied, while in the case where $p_{i}(q) \geq(\alpha-\varepsilon) / 2$, then $n^{*}\left(j_{0}\right)=n\left(j_{0}\right)$, and, therefore,

$$
\sum_{i=1}^{m} \sum_{q=n\left(j_{0}\right)}^{n^{*}\left(j_{0}\right)-1} p_{i}(q)=\sum_{i=1}^{m} \sum_{q=n\left(j_{0}\right)}^{n\left(j_{0}\right)-1} p_{i}(q)
$$

(by which we mean) $=0<\frac{\alpha-\varepsilon}{2}$,

$$
\sum_{i=1}^{m} \sum_{q=n\left(j_{0}\right)}^{n^{*}\left(j_{0}\right)} p_{i}(q)=\sum_{i=1}^{m} \sum_{q=n\left(j_{0}\right)}^{n\left(j_{0}\right)} p_{i}(q) \geq p_{i}\left(n\left(j_{0}\right)\right) \geq \frac{\alpha-\varepsilon}{2} .
$$

That is, in both cases (30) is satisfied.

Now, we will show that $\tau\left(n^{*}\left(j_{0}\right)\right) \leq n\left(j_{0}\right)-1$. Indeed, in the case where $p_{i}\left(n\left(j_{0}\right)\right) \geq(\alpha-\varepsilon) / 2$, since $n^{*}\left(j_{0}\right)=n\left(j_{0}\right)$, it is obvious that $\tau\left(n^{*}\left(j_{0}\right)\right)=\tau\left(n\left(j_{0}\right)\right) \leq n\left(j_{0}\right)-1$. In the case where $p_{i}\left(n\left(j_{0}\right)\right)<(\alpha-\varepsilon) / 2$, then $n^{*}\left(j_{0}\right)>n\left(j_{0}\right)$. Assume, for the sake of contradiction, that $\tau\left(n^{*}\left(j_{0}\right)\right)>n\left(j_{0}\right)-1$. Hence, $n\left(j_{0}\right) \leq \tau\left(n^{*}\left(j_{0}\right)\right) \leq n^{*}\left(j_{0}\right)-1$ and then

$$
\sum_{i=1}^{m} \sum_{q=\tau\left(n^{*}\left(j_{0}\right)\right)}^{n^{*}\left(j_{0}\right)-1} p_{i}(q) \leq \sum_{i=1}^{m} \sum_{q=n\left(j_{0}\right)}^{n^{*}\left(j_{0}\right)-1} p_{i}(q)<\frac{\alpha-\varepsilon}{2}
$$

which contradicts (28). Thus, in both cases, we have $\tau\left(n^{*}\left(j_{0}\right)\right) \leq n\left(j_{0}\right)-1$. Therefore

$$
\begin{aligned}
\sum_{i=1}^{m} \sum_{q=\tau\left(n^{*}\left(j_{0}\right)\right)}^{n\left(j_{0}\right)-1} p_{i}(q) & =\sum_{i=1}^{m} \sum_{q=\tau\left(n^{*}\left(j_{0}\right)\right)}^{n^{*}\left(j_{0}\right)-1} p_{i}(q)-\sum_{i=1}^{m} \sum_{q=n\left(j_{0}\right)}^{n^{*}\left(j_{0}\right)-1} p_{i}(q) \\
& >(\alpha-\varepsilon)-\frac{\alpha-\varepsilon}{2}=\frac{\alpha-\varepsilon}{2} .
\end{aligned}
$$

Summing up $\left(\mathrm{E}_{\mathrm{R}}\right)$ from $n\left(j_{0}\right)$ to $n^{*}\left(j_{0}\right)$, and using the fact that the function $x$ is decreasing and the function $\tau$ (as defined by (13)) is increasing, we have

$$
\begin{aligned}
x\left(n\left(j_{0}\right)\right) & =x\left(n^{*}\left(j_{0}\right)+1\right)+\sum_{i=1}^{m} \sum_{q=n\left(j_{0}\right)}^{n^{*}\left(j_{0}\right)} p_{i}(q) x\left(\tau_{i}(q)\right) \\
& \geq x\left(n^{*}\left(j_{0}\right)+1\right)+\sum_{i=1}^{m} \sum_{q=n\left(j_{0}\right)}^{n^{*}\left(j_{0}\right)} p_{i}(q) x(\tau(q)),
\end{aligned}
$$

or

$$
x\left(n\left(j_{0}\right)\right) \geq x\left(n^{*}\left(j_{0}\right)+1\right)+x\left(\tau\left(n^{*}\left(j_{0}\right)\right)\right) \sum_{i=1}^{m} \sum_{q=n\left(j_{0}\right)}^{n^{*}\left(j_{0}\right)} p_{i}(q)
$$

which, in view of (30), gives

$$
x\left(n\left(j_{0}\right)\right) \geq x\left(n^{*}\left(j_{0}\right)+1\right)+\frac{\alpha-\varepsilon}{2} x\left(\tau\left(n^{*}\left(j_{0}\right)\right)\right) .
$$

Summing up $\left(\mathrm{E}_{\mathrm{R}}\right)$ from $\tau\left(n^{*}\left(j_{0}\right)\right)$ to $n\left(j_{0}\right)-1$, and using the same arguments, we have

$$
\begin{aligned}
x\left(\tau\left(n^{*}\left(j_{0}\right)\right)\right) & =x\left(n\left(j_{0}\right)\right)+\sum_{i=1}^{m} \sum_{q=\tau\left(n^{*}\left(j_{0}\right)\right)}^{n\left(j_{0}\right)-1} p_{i}(q) x\left(\tau_{i}(q)\right) \\
& \geq x\left(n\left(j_{0}\right)\right)+\sum_{i=1}^{m} \sum_{q=\tau\left(n^{*}\left(j_{0}\right)\right)}^{n\left(j_{0}\right)-1} p_{i}(q) x(\tau(q)),
\end{aligned}
$$

or

$$
\begin{aligned}
& x\left(\tau\left(n^{*}\left(j_{0}\right)\right)\right) \\
& \quad \geq x\left(n\left(j_{0}\right)\right)+x\left(\tau\left(n\left(j_{0}\right)-1\right)\right) \sum_{i=1}^{m} \sum_{q=\tau\left(n^{*}\left(j_{0}\right)\right)}^{n\left(j_{0}\right)-1} p_{i}(q),
\end{aligned}
$$

which, in view of (34), gives

$$
x\left(\tau\left(n^{*}\left(j_{0}\right)\right)\right)>x\left(n\left(j_{0}\right)\right)+\frac{\alpha-\varepsilon}{2} x\left(\tau\left(n\left(j_{0}\right)-1\right)\right) .
$$

Combining inequalities (37) and (40), we obtain

$$
\begin{aligned}
x\left(n\left(j_{0}\right)\right)> & x\left(n^{*}\left(j_{0}\right)+1\right)+\frac{\alpha-\varepsilon}{2} \\
& \times\left[x\left(n\left(j_{0}\right)\right)+\frac{\alpha-\varepsilon}{2} x\left(\tau\left(n\left(j_{0}\right)-1\right)\right)\right],
\end{aligned}
$$

or

$$
\begin{aligned}
\left(1-\frac{\alpha-\varepsilon}{2}\right) x\left(n\left(j_{0}\right)\right) & \\
& >x\left(n^{*}\left(j_{0}\right)+1\right)+\left(\frac{\alpha-\varepsilon}{2}\right)^{2 .} x\left(\tau\left(n\left(j_{0}\right)-1\right)\right) .
\end{aligned}
$$

Thus

$$
x\left(n\left(j_{0}\right)\right)>\frac{(\alpha-\varepsilon)^{2}}{2[2-(\alpha-\varepsilon)]} x\left(\tau\left(n\left(j_{0}\right)-1\right)\right) .
$$

In view of (43), inequality (42) gives

$$
\begin{aligned}
x\left(n\left(j_{0}\right)\right)> & \frac{(\alpha-\varepsilon)^{2} /(2[2-(\alpha-\varepsilon)])}{1-((\alpha-\varepsilon) / 2)} x\left(\tau\left(n^{*}\left(j_{0}\right)\right)\right) \\
& +\frac{(\alpha-\varepsilon)^{2}}{2[2-(\alpha-\varepsilon)]} x\left(\tau\left(n\left(j_{0}\right)-1\right)\right)
\end{aligned}
$$

which, in view of (40) becomes

$$
\begin{aligned}
x\left(n\left(j_{0}\right)\right)> & \frac{(\alpha-\varepsilon)^{2} /(2[2-(\alpha-\varepsilon)])}{1-((\alpha-\varepsilon) / 2)} \\
& \times\left[x\left(n\left(j_{0}\right)\right)+\frac{\alpha-\varepsilon}{2} x\left(\tau\left(n\left(j_{0}\right)-1\right)\right)\right] \\
& +\frac{(\alpha-\varepsilon)^{2}}{2[2-(\alpha-\varepsilon)]} x\left(\tau\left(n\left(j_{0}\right)-1\right)\right) .
\end{aligned}
$$


Thus

$$
\frac{x\left(n\left(j_{0}\right)\right)}{x\left(\tau\left(n\left(j_{0}\right)-1\right)\right)}>\frac{(\alpha-\varepsilon)^{2}}{4[1-(\alpha-\varepsilon)]}
$$

or

$$
\frac{x\left(n\left(j_{0}\right)+1\right)}{x\left(\tau\left(n\left(j_{0}\right)\right)\right)}>\frac{(\alpha-\varepsilon)^{2}}{4[1-(\alpha-\varepsilon)]} .
$$

Hence,

$$
\liminf _{j_{0} \rightarrow \infty} \frac{x\left(n\left(j_{0}\right)+1\right)}{x\left(\tau\left(n\left(j_{0}\right)\right)\right)} \geq \frac{(\alpha-\varepsilon)^{2}}{4[1-(\alpha-\varepsilon)]}
$$

which, for arbitrarily small values of $\varepsilon$, implies (25).

The proof of the lemma is complete.

Theorem 8. Assume that (1) holds, the sequences $\tau_{i}$ are increasing for all $i \in\{1, \ldots, m\}$ and $\tau$ is defined by (13). Suppose also that for each $i \in\{1, \ldots, m\}$ there exists a sequence $\left\{n_{i}(j)\right\}_{j \in \mathbb{N}}$ such that $\lim _{j \rightarrow \infty} n_{i}(j)=\infty$, (12) and define $\alpha$ by (24), where $n(j)=\min \left\{n_{i}(j): 1 \leq i \leq m\right\}$.

If $0<\alpha<1$, and

$$
\limsup _{j \rightarrow \infty} \sum_{i=1}^{m} \sum_{q=\tau(n(j))}^{n(j)} p_{i}(q)>1-\frac{\alpha^{2}}{4(1-\alpha)}
$$

then all solutions of $\left(\mathrm{E}_{\mathrm{R}}\right)$ oscillate.

Proof. Assume, for the sake of contradiction, that $\{x(n)\}_{n \geq-w}$ is an eventually positive solution of $\left(E_{R}\right)$. Then there exists $j_{0} \in \mathbb{N}$ such that

$$
\begin{array}{r}
p_{k}(n) \geq 0 \quad \forall n \in \bigcap_{k=1}^{m}\left[\tau\left(\tau\left(n_{k}\left(j_{0}\right)\right)\right), n_{k}\left(j_{0}\right)\right] \cap \mathbb{N}, \\
1 \leq k \leq m, \\
x\left(\tau_{k}(n)\right)>0 \quad \forall n \in \bigcap_{k=1}^{m}\left[\tau\left(\tau\left(n_{k}\left(j_{0}\right)\right)\right), n_{k}\left(j_{0}\right)\right] \cap \mathbb{N}, \\
1 \leq k \leq m .
\end{array}
$$

Therefore, by $\left(\mathrm{E}_{\mathrm{R}}\right)$ we have

$$
x(n+1)-x(n)=-\sum_{i=1}^{m} p_{i}(n) x\left(\tau_{i}(n)\right) \leq 0,
$$

for every $n \in \bigcap_{k=1}^{m}\left[\tau\left(\tau\left(n_{k}\left(j_{0}\right)\right)\right), n_{k}\left(j_{0}\right)\right] \cap \mathbb{N}$. This guarantees that the sequence $x$ is decreasing on $\bigcap_{k=1}^{m}\left[\tau\left(\tau\left(n_{k}\left(j_{0}\right)\right)\right)\right.$, $\left.n_{k}\left(j_{0}\right)\right] \cap \mathbb{N}$.
Summing up $\left(\mathrm{E}_{\mathrm{R}}\right)$ from $\tau\left(n\left(j_{0}\right)\right)$ to $n\left(j_{0}\right)$, and using the fact that the function $x$ is decreasing and the function $\tau$ (as defined by (13)) is increasing, we obtain

$$
\begin{aligned}
x\left(\tau\left(n\left(j_{0}\right)\right)\right)= & x\left(n\left(j_{0}\right)+1\right)+\sum_{i=1}^{m} \sum_{q=\tau\left(n\left(j_{0}\right)\right)}^{n\left(j_{0}\right)} p_{i}(q) x\left(\tau_{i}(q)\right) \\
\geq & x\left(n\left(j_{0}\right)+1\right)+x\left(\tau\left(n\left(j_{0}\right)\right)\right) \\
& \times \sum_{i=1}^{m} \sum_{q=\tau\left(n\left(j_{0}\right)\right)}^{n\left(j_{0}\right)} p_{i}(q) .
\end{aligned}
$$

Consequently,

$$
\sum_{i=1}^{m} \sum_{q=\tau\left(n\left(j_{0}\right)\right)}^{n\left(j_{0}\right)} p_{i}(q) \leq 1-\frac{x\left(n\left(j_{0}\right)+1\right)}{x\left(\tau\left(n\left(j_{0}\right)\right)\right)}
$$

which gives

$$
\limsup _{j_{0} \rightarrow \infty} \sum_{i=1}^{m} \sum_{q=\tau\left(n\left(j_{0}\right)\right)}^{n\left(j_{0}\right)} p_{i}(q) \leq 1-\liminf _{j_{0} \rightarrow \infty} \frac{x\left(n\left(j_{0}\right)+1\right)}{x\left(\tau\left(n\left(j_{0}\right)\right)\right)} .
$$

Assume that $0<\alpha<1$ and (49) holds. Then by Lemma 7, inequality (25) is fulfilled, and so (54) leads to

$$
\limsup _{j_{0} \rightarrow \infty} \sum_{i=1}^{m} \sum_{q=\tau\left(n\left(j_{0}\right)\right)}^{n\left(j_{0}\right)} p_{i}(q) \leq 1-\frac{\alpha^{2}}{4(1-\alpha)},
$$

which contradicts condition (49).

The proof of the theorem is complete.

\section{Advanced Equations}

Oscillation of all solutions of $\left(\mathrm{E}_{\mathrm{A}}\right)$ is described by the theorem below. Note that the proof is an easy modification of the proof of Theorem 8 and hence is omitted.

Theorem 9. Assume (2) holds, the sequences $\sigma_{i}$ are increasing for all $i \in\{1, \ldots, m\}$ and $\sigma$ is defined by (16). Suppose also that for each $i \in\{1, \ldots, m\}$ there exists a sequence $\left\{n_{i}(j)\right\}_{j \in \mathbb{N}}$ such that $\lim _{j \rightarrow \infty} n_{i}(j)=\infty$, (15) and

$$
\alpha:=\liminf _{j \rightarrow \infty} \sum_{i=1}^{m} \sum_{q=n(j)+1}^{\sigma(n(j))} p_{i}(q),
$$

where $n(j)=\max \left\{n_{i}(j): 1 \leq i \leq m\right\}$.

If $0<\alpha<1$ and

$$
\limsup _{j \rightarrow \infty} \sum_{i=1}^{m} \sum_{q=n(j)}^{\sigma(n(j))} p_{i}(q)>1-\frac{\alpha^{2}}{4(1-\alpha)},
$$

then all solutions of $\left(\mathrm{E}_{\mathrm{A}}\right)$ oscillate.

Remark 10. When $\alpha \rightarrow 0$, then the conditions (49) and (57) reduce to the conditions (14) and (17), respectively. However the improvement is clear when $\alpha \rightarrow 1 / e$. The lower bound in (49) and (57) is 0.946475699 . That is, when $0<\alpha<1 / e$, our conditions (49) and (57) essentially improve (14) and (17). 


\section{Examples}

The significance of the results is illustrated in the following examples.

Example 1. Consider the retarded difference equation

$$
\begin{aligned}
\Delta x(n) & +p_{1}(n) x(n-2)+p_{2}(n) x(n-3) \\
& +p_{3}(n) x(n-4)=0, \quad n \in \mathbb{N}_{0},
\end{aligned}
$$

where $p_{1}(n), p_{2}(n)$, and $p_{3}(n)$ are oscillating coefficients, as shown in Figure 1.

In view of (13), it is obvious that $\tau(n)=n-2$. Observe that for

$$
n_{1}(j)=20 j+9, \quad j \in \mathbb{N}
$$

we have $p_{1}(n)>0$ for every $n \in A$, where

$$
\begin{aligned}
A & =\bigcup_{j \in \mathbb{N}}\left[\tau\left(\tau\left(n_{1}(j)\right)\right), n_{1}(j)\right] \cap \mathbb{N} \\
& =\bigcup_{j \in \mathbb{N}}[20 j+5,20 j+9] \cap \mathbb{N} .
\end{aligned}
$$

For

$$
n_{2}(j)=20 j+8, \quad j \in \mathbb{N}
$$

we have $p_{2}(n)>0$ for every $n \in B$, where

$$
\begin{aligned}
B & =\bigcup_{j \in \mathbb{N}}\left[\tau\left(\tau\left(n_{2}(j)\right)\right), n_{2}(j)\right] \cap \mathbb{N} \\
& =\bigcup_{j \in \mathbb{N}}[20 j+4,20 j+8] \cap \mathbb{N}
\end{aligned}
$$

and, for

$$
n_{3}(j)=20 j+9, \quad j \in \mathbb{N}
$$

we have $p_{3}(n) \geq 0$ for every $n \in C$, where

$$
\begin{aligned}
C & =\bigcup_{j \in \mathbb{N}}\left[\tau\left(\tau\left(n_{3}(j)\right)\right), n_{3}(j)\right] \cap \mathbb{N} \\
& =\bigcup_{j \in \mathbb{N}}[20 j+5,20 j+9] \cap \mathbb{N} .
\end{aligned}
$$

Therefore,

$$
\begin{array}{r}
p_{1}(n)>0, \quad p_{2}(n)>0, \quad p_{3}(n) \geq 0 \\
\forall n \in A \cap B \cap C \\
=\bigcup_{j \in \mathbb{N}}[20 j+5,20 j+8] \cap \mathbb{N} \neq \emptyset .
\end{array}
$$

Observe that

$$
n(j)=\min \left\{n_{i}(j): 1 \leq i \leq 3\right\}=20 j+8, \quad j \in \mathbb{N} .
$$
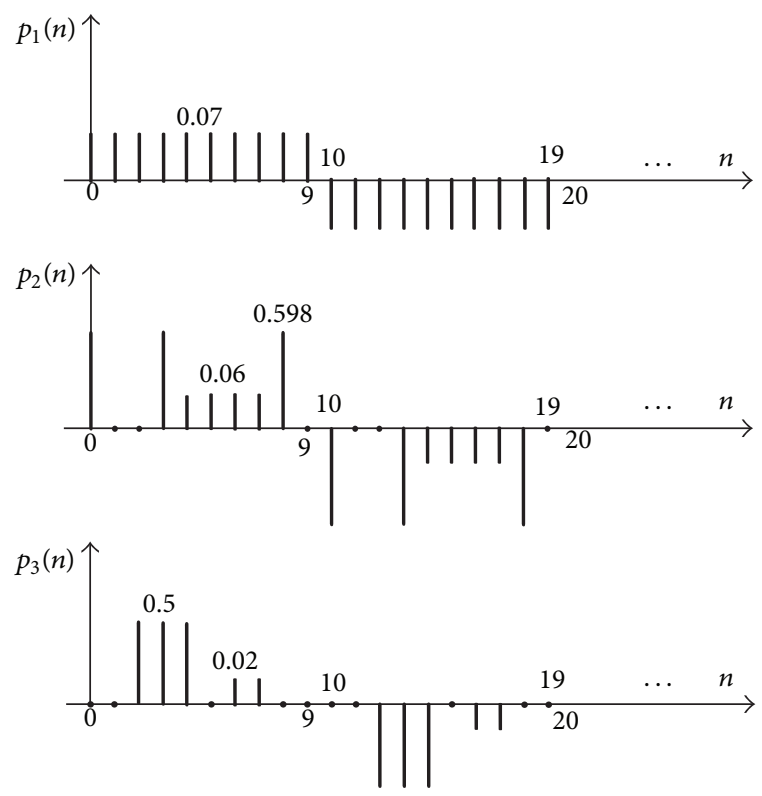

Figure 1

Now,

$\alpha=\liminf _{j \rightarrow \infty} \sum_{i=1}^{m} \sum_{q=\tau(n(j))}^{n(j)-1} p_{i}(q)$

$$
\begin{aligned}
& =\liminf _{j \rightarrow \infty}\left[\sum_{q=20 j+6}^{20 j+7} p_{1}(q)+\sum_{q=20 j+6}^{20 j+7} p_{2}(q)+\sum_{q=20 j+6}^{20 j+7} p_{3}(q)\right] \\
& =2 \cdot \frac{7}{100}+2 \cdot \frac{6}{100}+2 \cdot \frac{2}{100}=0.3
\end{aligned}
$$

$\limsup _{j \rightarrow \infty} \sum_{i=1}^{2} \sum_{q=\tau(n(j))}^{n(j)} p_{i}(q)$

$$
\begin{aligned}
& =\limsup _{j \rightarrow \infty}\left[\sum_{q=20 j+6}^{20 j+8} p_{1}(q)+\sum_{q=20 j+6}^{20 j+8} p_{2}(q)+\sum_{q=20 j+6}^{20 j+8} p_{3}(q)\right] \\
& =3 \cdot \frac{7}{100}+2 \cdot \frac{6}{100}+\frac{598}{1000}+2 \cdot \frac{2}{100}=0.968 .
\end{aligned}
$$

Observe that

$$
0.968>1-\frac{\alpha^{2}}{4(1-\alpha)} \simeq 0.967857142
$$

that is, condition (49) of Theorem 8 is satisfied and, therefore, all solutions of equation (58) oscillate.

On the other hand, 
Observe that $p_{1}(n)>0$ for every $n \in A^{\prime}=A, p_{2}(n) \geq 0$ for every $n \in B^{\prime}$, where

$$
\begin{aligned}
B^{\prime} & =\bigcup_{j \in \mathbb{N}}\left[\tau_{2}\left(\tau_{2}\left(n_{2}(j)\right)\right), n_{2}(j)\right] \cap \mathbb{N} \\
& =\bigcup_{j \in \mathbb{N}}[20 j+2,20 j+8] \cap \mathbb{N},
\end{aligned}
$$

and $p_{3}(n) \geq 0$ for every $n \in C^{\prime}$, where

$$
\begin{aligned}
C^{\prime} & =\bigcup_{j \in \mathbb{N}}\left[\tau_{3}\left(\tau_{3}\left(n_{3}(j)\right)\right), n_{3}(j)\right] \cap \mathbb{N} \\
& =\bigcup_{j \in \mathbb{N}}[20 j+1,20 j+9] \cap \mathbb{N} .
\end{aligned}
$$

Therefore,

$$
\begin{array}{r}
p_{1}(n)>0, \quad p_{2}(n)>0, \quad p_{3}(n) \geq 0 \\
\forall n \in A^{\prime} \cap B^{\prime} \cap C^{\prime} \\
=\bigcup_{j \in \mathbb{N}}[20 j+5,20 j+8] \cap \mathbb{N} \neq \emptyset .
\end{array}
$$

Also,

$$
\begin{aligned}
& \liminf _{j \rightarrow \infty} \sum_{i=1}^{2} \sum_{q=\tau_{i}\left(n_{i}(j)\right)}^{n_{i}(j)-1} p_{i}(q) \\
& =\liminf _{j \rightarrow \infty}\left[\sum_{q=20 j+7}^{20 j+8} p_{1}(q)+\sum_{q=20 j+5}^{20 j+7} p_{2}(q)+\sum_{q=20 j+5}^{20 j+8} p_{3}(q)\right] \\
& =2 \cdot \frac{7}{100}+3 \cdot \frac{6}{100}+2 \cdot \frac{2}{100}=0.36<\frac{1}{e} .
\end{aligned}
$$

Therefore none of the conditions (14) and (20) is satisfied.

Example 2. Consider the advanced difference equation

$$
\nabla x(n)-p_{1}(n) x(n+1)-p_{2}(n) x(n+2)=0, \quad n \in \mathbb{N},
$$

where $p_{1}(n)$ and $p_{2}(n)$ are oscillating coefficients, as shown in Figure 2.

In view of (16), it is obvious that $\sigma(n)=n+1$. Observe that for

$$
n_{1}(j)=16 j, \quad j \in \mathbb{N},
$$

we have $p_{1}(n) \geq 0$ for every $n \in A$, where

$$
A=\bigcup_{j \in \mathbb{N}}\left[n_{1}(j), \sigma\left(\sigma\left(n_{1}(j)\right)\right)\right] \cap \mathbb{N}=\bigcup_{j \in \mathbb{N}}[16 j, 16 j+2] \cap \mathbb{N} .
$$

Also, for

$$
n_{2}(j)=16 j+1, \quad j \in \mathbb{N},
$$
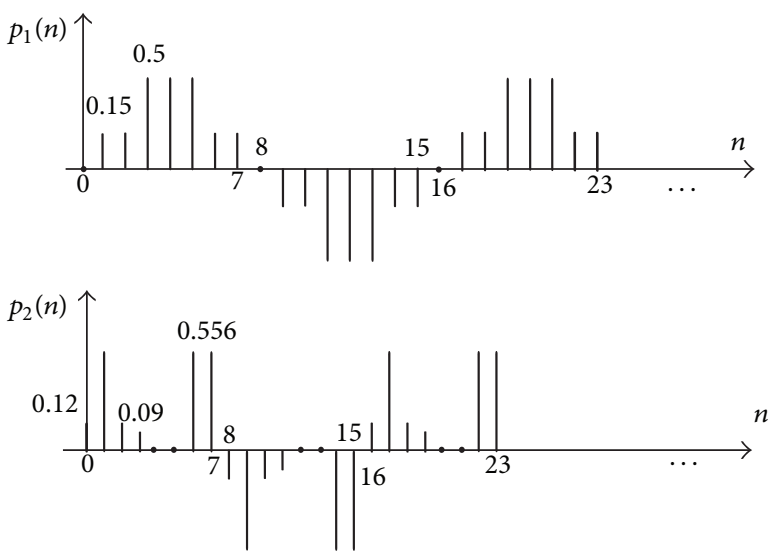

FiguRe 2

we have $p_{2}(n)>0$ for every $n \in B$, where

$$
\begin{aligned}
B & =\bigcup_{j \in \mathbb{N}}\left[n_{2}(j), \sigma\left(\sigma\left(n_{2}(j)\right)\right)\right] \cap \mathbb{N} \\
& =\bigcup_{j \in \mathbb{N}}[16 j+1,16 j+3] \cap \mathbb{N} .
\end{aligned}
$$

Therefore,

$$
\begin{aligned}
& p_{1}(n)>0, \quad p_{2}(n)>0 \\
& \forall n \in A \cap B=\bigcup_{j \in \mathbb{N}}[16 j+1,16 j+2] \cap \mathbb{N} \neq \emptyset .
\end{aligned}
$$

Observe that

$$
n(j)=\max \left\{n_{i}(j): 1 \leq i \leq 2\right\}=16 j+1, \quad j \in \mathbb{N} .
$$

Now

$$
\begin{aligned}
\alpha & =\liminf _{j \rightarrow \infty} \sum_{i=1}^{2} \sum_{q=n(j)+1}^{\sigma(n(j))} p_{i}(q) \\
& =\liminf _{j \rightarrow \infty}\left[\sum_{q=16 j+2}^{16 j+2} p_{1}(q)+\sum_{q=16 j+2}^{16 j+2} p_{2}(q)\right] \\
& =\frac{15}{100}+\frac{12}{100}=0.27 .
\end{aligned}
$$

Also

$$
\begin{aligned}
& \limsup _{j \rightarrow \infty} \sum_{i=1}^{2} \sum_{q=n(j)}^{\sigma(n(j))} p_{i}(q) \\
& \quad=\limsup _{j \rightarrow \infty}\left[\sum_{q=16 j+1}^{16 j+2} p_{1}(q)+\sum_{q=16 j+1}^{16 j+2} p_{2}(q)\right] \\
& =2 \cdot \frac{15}{100}+\frac{12}{100}+\frac{556}{1000}=0.976
\end{aligned}
$$


Observe that

$$
0.976>1-\frac{\alpha^{2}}{4(1-\alpha)} \simeq 0.975034246 ;
$$

that is, condition (57) of Theorem 9 is satisfied and, therefore, all solutions of equation (74) oscillate.

On the other hand,

$$
0.976<1
$$

Observe that $p_{1}(n) \geq 0$ for every $n \in A^{\prime}=A$ and $p_{2}(n) \geq 0$ for every $n \in B^{\prime}$, where

$$
\begin{aligned}
B^{\prime} & =\bigcup_{j \in \mathbb{N}}\left[n_{2}(j), \sigma_{2}\left(\sigma_{2}\left(n_{2}(j)\right)\right)\right] \cap \mathbb{N} \\
& =\bigcup_{j \in \mathbb{N}}[16 j+1,16 j+5] \cap \mathbb{N} .
\end{aligned}
$$

Therefore,

$$
\begin{gathered}
p_{1}(n)>0, \quad p_{2}(n)>0 \\
\forall n \in A^{\prime} \cap B^{\prime} \\
=\bigcup_{j \in \mathbb{N}}[16 j+1,16 j+2] \neq \emptyset .
\end{gathered}
$$

Also,

$$
\begin{aligned}
& \liminf _{j \rightarrow \infty} \sum_{i=1}^{m} \sum_{q=n_{i}(j)+1}^{\sigma_{i}\left(n_{i}(j)\right)} p_{i}(q) \\
& =\liminf _{j \rightarrow \infty}\left[\sum_{q=16 j+1}^{16 j+1} p_{1}(q)+\sum_{q=16 j+2}^{16 j+3} p_{2}(q)\right] \\
& =\frac{15}{100}+\frac{12}{100}+\frac{9}{100}=0.36<\frac{1}{e} .
\end{aligned}
$$

Therefore none of the conditions (17) and (23) is satisfied.

\section{Conflict of Interests}

The authors declare that there is no conflict of interests regarding the publication of this paper.

\section{Acknowledgment}

The authors would like to thank the referees for the constructive remarks which improved the presentation of the paper.

\section{References}

[1] N. Fukagai and T. Kusano, "Oscillation theory of first order functional-differential equations with deviating arguments," Annali di Matematica Pura ed Applicata, vol. 136, pp. 95-117, 1984.

[2] G. E. Chatzarakis and I. P. Stavroulakis, "Oscillations of difference equations with general advanced argument," Central European Journal of Mathematics, vol. 10, no. 2, pp. 807-823, 2012.
[3] R. P. Agarwal, L. Berezansky, E. Braverman, and A. Domoshnitsky, Nonoscillation Theory of Functional Differential Equations with Applications, Springer, New York, NY, USA, 2012.

[4] L. Berezansky and E. Braverman, "On existence of positive solutions for linear difference equations with several delays," Advances in Dynamical Systems and Applications, vol. 1, no. 1, pp. 29-47, 2006.

[5] G. E. Chatzarakis, R. Koplatadze, and I. P. Stavroulakis, "Optimal oscillation criteria for first order difference equations with delay argument," Pacific Journal of Mathematics, vol. 235, no. 1, pp. 15-33, 2008.

[6] G. E. Chatzarakis, R. Koplatadze, and I. P. Stavroulakis, "Oscillation criteria of first order linear difference equations with delay argument," Nonlinear Analysis: Theory, Methods \& Applications, vol. 68, no. 4, pp. 994-1005, 2008.

[7] G. E. Chatzarakis, T. Kusano, and I. P. Stavroulakis, "Oscillation conditions for difference equations with several variable arguments," Mathematica Bohemica. In press.

[8] G. E. Chatzarakis, J. Manojlovic, S. Pinelas, and I. P. Stavroulakis, "Oscillation criteria of difference equations with several deviating arguments," Yokohama Mathematical Journal. In press.

[9] G. E. Chatzarakis, S. Pinelas, and I. P. Stavroulakis, "Oscillations of difference equations with several deviated arguments," Aequationes Mathematicae. In press.

[10] F. M. Dannan and S. N. Elaydi, "Asymptotic stability of linear difference equations of advanced type," Journal of Computational Analysis and Applications, vol. 6, no. 2, pp. 173-187, 2004.

[11] L. Berezansky, Y. Domshlak, and E. Braverman, "On oscillation properties of delay differential equations with positive and negative coefficients," Journal of Mathematical Analysis and Applications, vol. 274, no. 1, pp. 81-101, 2002.

[12] A. Domoshnitsky, M. Drakhlin, and I. P. Stavroulakis, "Distribution of zeros of solutions to functional equations," Mathematical and Computer Modelling, vol. 42, no. 1-2, pp. 193-205, 2005.

[13] M. K. Grammatikopoulos, R. Koplatadze, and I. P. Stavroulakis, "On the oscillation of solutions of first order differential equations with retarded arguments," Georgian Mathematical Journal, vol. 10, no. 1, pp. 63-76, 2003.

[14] H. Khatibzadeh, "An oscillation criterion for a delay difference equation," Computers \& Mathematics with Applications, vol. 57, no. 1, pp. 37-41, 2009.

[15] R. G. Koplatadze and T. A. Chanturiya, "Oscillating and monotone solutions of first-order differential equations with deviating argument," Differentsial' nye Uravneniya, vol. 18, no. 8, p. 1463-1465, 1472, 1982.

[16] G. Ladas and I. P. Stavroulakis, "Oscillations caused by several retarded and advanced arguments," Journal of Differential Equations, vol. 44, no. 1, pp. 134-152, 1982.

[17] X. Li and D. Zhu, "Oscillation and nonoscillation of advanced differential equations with variable coefficients," Journal of Mathematical Analysis and Applications, vol. 269, no. 2, pp. 462$488,2002$.

[18] X. Li and D. Zhu, "Oscillation of advanced difference equations with variable coefficients," Annals of Differential Equations, vol. 18, no. 3, pp. 254-263, 2002.

[19] H. Onose, "Oscillatory properties of the first-order differential inequalities with deviating argument," Funkcialaj Ekvacioj, vol. 26, no. 2, pp. 189-195, 1983.

[20] B. G. Zhang, "Oscillation of the solutions of the first-order advanced type differential equations," Science Exploration, vol. 2, no. 3, pp. 79-82, 1982. 
[21] M. Bohner, G. E. Chatzarakis, and I. P. Stavroulakis, "Oscillation criteria for difference equations with several oscillating coeffcients," In press.

[22] A. Domoshnitsky, "Maximum principles, boundary value problems and stability for first order delay equations with oscillating coefficient," International Journal of Qualitative Theory of Differential Equations and Applications, vol. 3, no. 1-2, pp. 33-42, 2009.

[23] M. Bohner, G. E. Chatzarakis, and I. P. Stavroulakis, "Qualitative behavior of solutions of difference equations with several oscillating coefficients," Arabian Journal of Mathematics, vol. 3, no. 1, pp. 1-13, 2014.

[24] M. R. Kulenović and M. K. Grammatikopoulos, "First order functional-differential inequalities with oscillating coefficients," Nonlinear Analysis: Theory, Methods \& Applications, vol. 8, no. 9, pp. 1043-1054, 1984.

[25] G. Ladas, Y. G. Sficas, and I. P. Stavroulakis, "Functionaldifferential inequalities and equations with oscillating coefficients," in Trends in Theory and Practice of Nonlinear Differential Equations, vol. 90 of Lecture Notes in Pure and Applied Mathematics, pp. 277-284, Dekker, New York, NY, USA, 1984.

[26] X. Li, D. Zhu, and H. Wang, "Oscillation for advanced differential equations with oscillating coefficients," International Journal of Mathematics and Mathematical Sciences, no. 33, pp. 21092118, 2003.

[27] Q. Chuanxi, G. Ladas, and J. Yan, "Oscillation of difference equations with oscillating coefficients," Radovi Matematički, vol. 8, no. 1, pp. 55-65, 1992.

[28] X.-H. Tang and S. S. Cheng, "An oscillation criterion for linear difference equations with oscillating coefficients," Journal of Computational and Applied Mathematics, vol. 132, no. 2, pp. 319329, 2001.

[29] X. Tang, "Oscillation of first order delay differential equations with oscillating coefficients," Applied Mathematics B, vol. 15, no. 3, pp. 252-258, 2000.

[30] W. P. Yan and J. R. Yan, "Comparison and oscillation results for delay difference equations with oscillating coefficients," International Journal of Mathematics and Mathematical Sciences, vol. 19, no. 1, pp. 171-176, 1996.

[31] J. S. Yu and X. H. Tang, "Sufficient conditions for the oscillation of linear delay difference equations with oscillating coefficients," Journal of Mathematical Analysis and Applications, vol. 250, no. 2, pp. 735-742, 2000.

[32] J. S. Yu, B. G. Zhang, and X. Z. Qian, "Oscillations of delay difference equations with oscillating coefficients," Journal of Mathematical Analysis and Applications, vol. 177, no. 2, pp. 432444, 1993. 


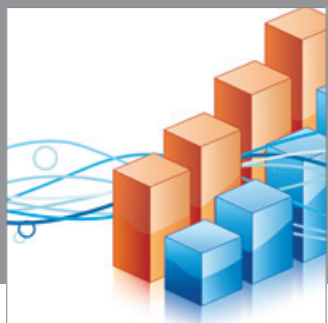

Advances in

Operations Research

mansans

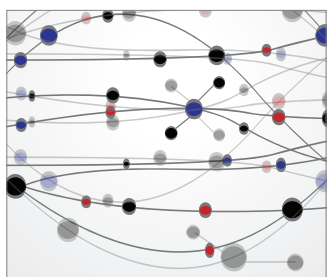

The Scientific World Journal
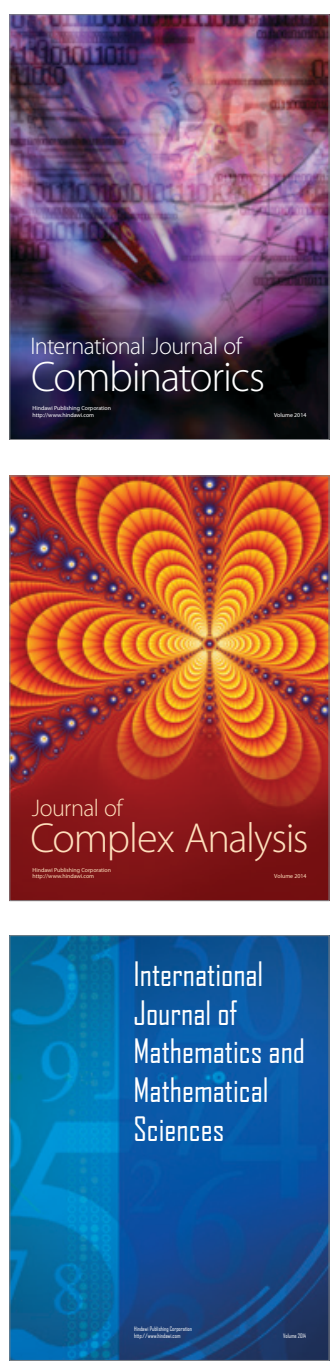
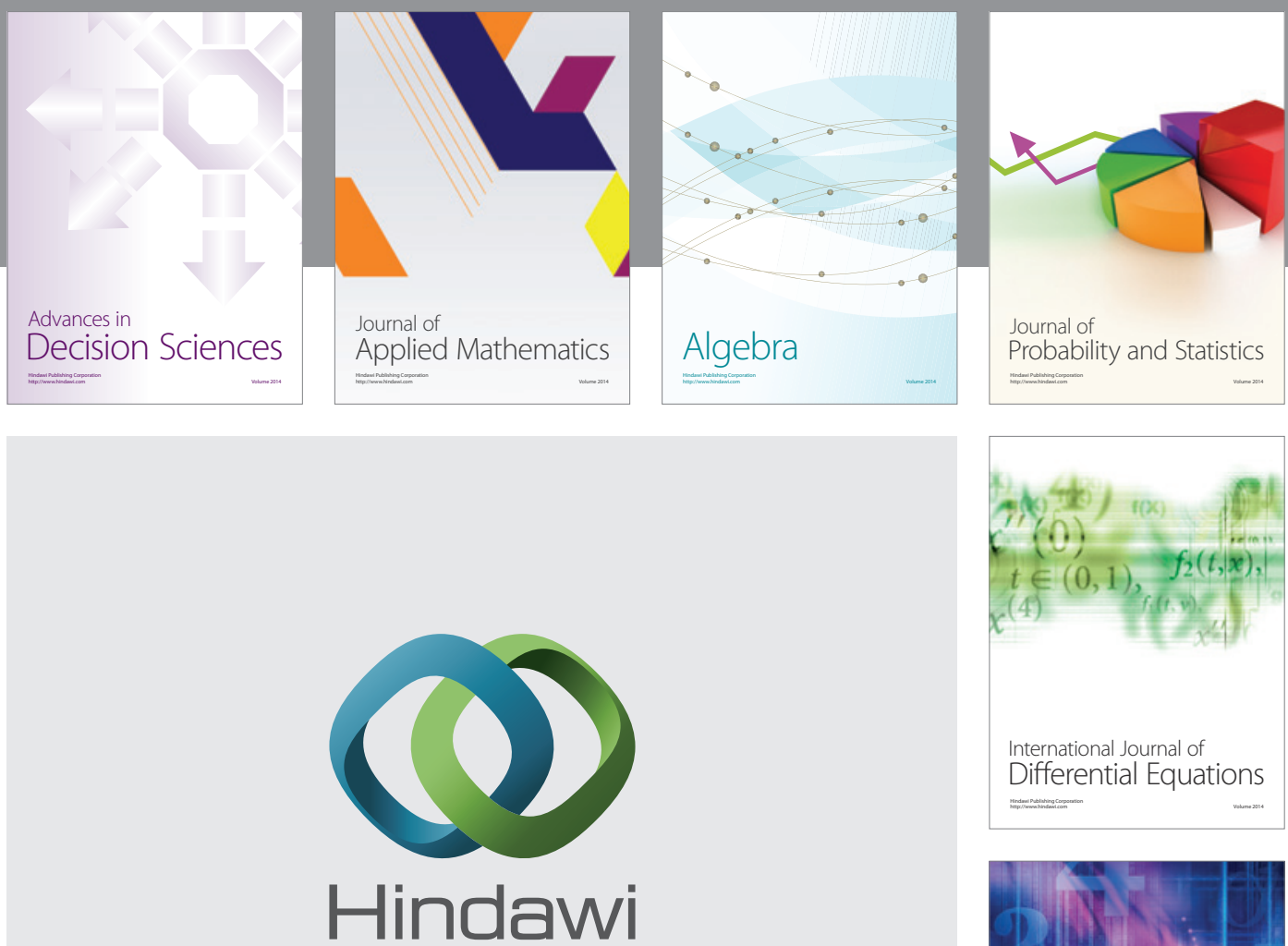

Submit your manuscripts at http://www.hindawi.com
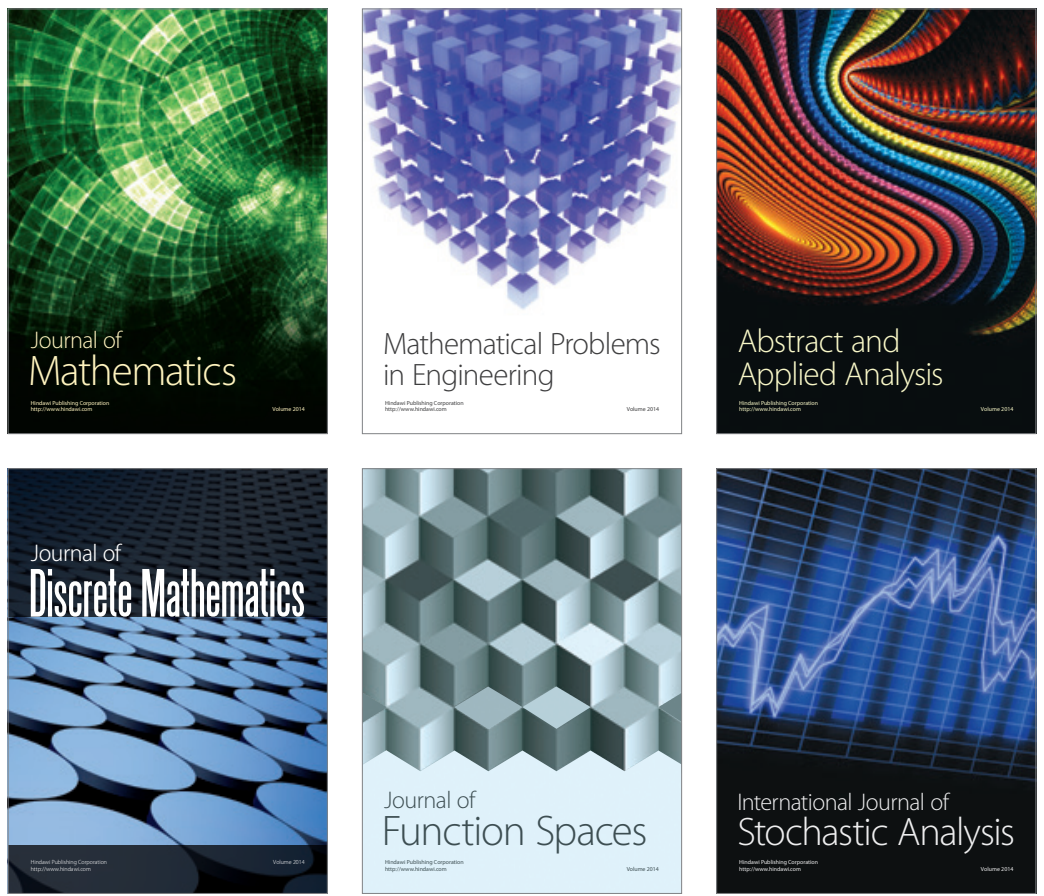

Journal of

Function Spaces

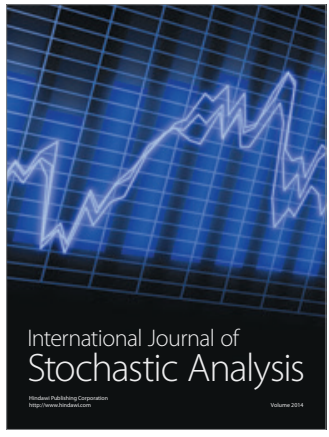

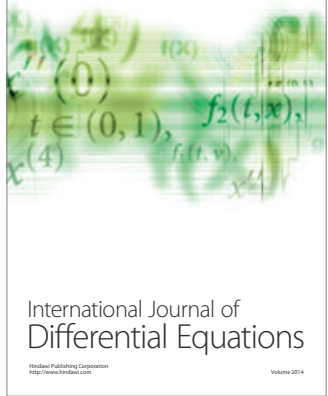
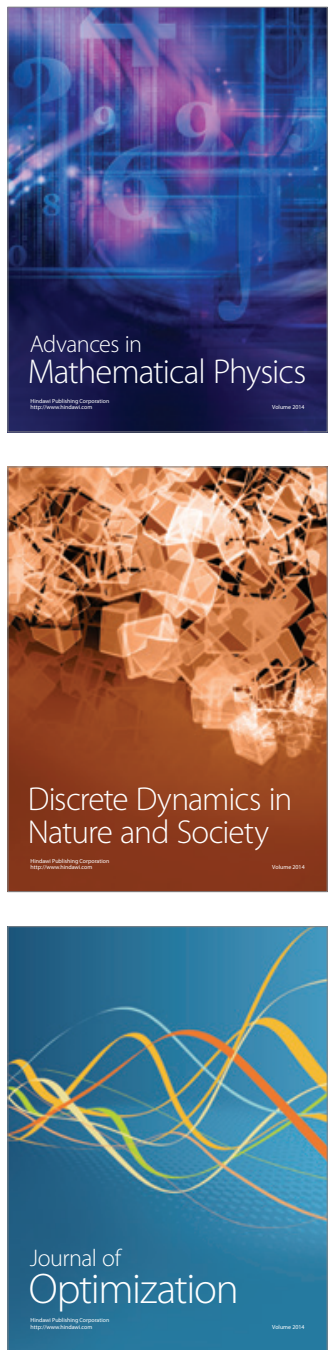